\title{
ADAPTAÇÃO TRANSCULTURAL DE UM INSTRUMENTO DE AVALIAÇÃO DE FERIDAS
}

\author{
Elaine Aparecida Rocha Domingues', Maiúme Roana Ferreira de Carvalho², \\ Uiara Aline de Oliveira Kaizer ${ }^{3}$
}

Objetivo: traduzir e adaptar culturalmente o instrumento de Avaliação da Cicatrização de Feridas Crônicas para a língua portuguesa do Brasil. Método: estudo quantitativo, tipo metodológico, seguindo as etapas tradução, síntese das traduções, retrotradução, revisão por um comitê de especialistas e pré-teste. A pesquisa foi realizada nas Unidades de Saúde de Itajubá e Três Corações - Minas Gerais com amostra de 30 enfermeiros estomaterapeutas e/ou com prática clínica no tratamento de feridas. Foi avaliada a validade de conteúdo por meio do cálculo do Índice de Validade de Conteúdo e praticabilidade do instrumento. Resultado: o instrumento apresentou-se como de fácil aplicação e compreensibilidade demonstrando ser aplicável na cultura brasileira. Quanto à praticabilidade, o tempo médio de preenchimento do instrumento foi de três minutos. O instrumento final, com alterações sugeridas pelos juízes, foi encaminhado e aprovado pelo autor. Conclusão: originou-se um instrumento aplicável à realidade brasileira.

DESCRITORES: Ferimentos e lesões; Validade dos testes; Tradução; Pesquisa metodológica em Enfermagem; Úlcera de perna.

\section{CROSS-CULTURAL ADAPTATION OF A WOUND ASSESSMENT INSTRUMENT}

Objective: To translate and culturally adapt a chronic wound healing assessment instrument into Brazilian Portuguese. Method: A methodological quantitative study was carried out based on the following stages: translation, translation synthesis, back-translation, revision by an expert committee, and pre-test. The research was carried out in the healthcare units of the cities of Itajubá and Três Corações, in the state of Minas Gerais, with a sample made up of 30 stomal therapy nurses and nurses with clinical practice in wound care. Content validity was evaluated by means of calculation of the content validity index and the instrument's feasibility. Result: The instrument was considered of easy application and understandability, indicating that it can be used in the Brazilian culture. Regarding feasibility, the instrument's average filling time was three minutes. The final instrument, with alterations suggested by the experts, was sent to the author and approved. Conclusion: An instrument applicable to the Brazilian reality was created. DESCRIPTORS: Wounds and Injuries; Reproducibility of Results; Translation; Nursing Methodology Research; Leg Ulcers.

\section{ADAPTACIÓN TRANSCULTURAL DE UN INSTRUMENTO DE EVALUACIÓN DE HERIDAS}

Objetivo: Traducir y adaptar culturalmente el instrumento de Evaluación de la Cicatrización de Heridas Crónicas al portugués brasileño. Método: Estudio cuantitativo, tipo metodológico, siguiéndose las etapas de traducción, síntesis de traducciones, retrotraducción, revisión por comité de expertos y prueba piloto. Investigación realizada en las Unidades de Salud de Itajubá y Três Corações - Minas Gerais. Muestra de 30 enfermeros estomaterapeutas y/o con práctica clínica en el tratamiento de heridas. Fue evaluada la validez de contenido mediante el cálculo del Índice de Validez de Contenido y viabilidad del instrumento. Resultado: El instrumento se presentó como de fácil aplicación y comprensibilidad, demostrándose aplicable a la cultura brasileña. Respecto a la viabilidad, el tiempo promedio de completado del instrumento fue de tres minutos. La versión final, con cambios sugeridos por los expertos, fue remitida y aprobada por el autor. Conclusión: Se dio origen a un instrumento aplicable a la realidad brasileña. DESCRIPTORES: Heridas y Lesiones; Reproductibilidad de los Resultados; Traducción; Investigación Metodológica en Enfermería; Úlcera de la Pierna.

${ }^{1}$ Enfermeira, Mestra em Ciências da Saúde. Coordenadora do Curso de Enfermagem da Universidade Vale do Rio Verde-UninCor. Três Corações. Minas Gerais, Brasil.

²Enfermeira, Mestra em Ciências Aplicadas à Saúde. Supervisora de estágio do Curso de Enfermagem da Universidade Vale do Rio Verde-UninCor. Três Corações. Minas Gerais, Brasil.

${ }^{3}$ Enfermeira, Mestra em Ciências da Saúde. Supervisora do Ambulatório de Feridas e Pé Diabético e Programa Municipal de Controle da Hanseníase da Prefeitura de Sorocaba, Sorocaba, São Paulo. Brasil.

\section{Autor Correspondente}

Maiúme Roana Ferreira de Carvalho.

Universidade Vale do Rio Verde-UninCor.

Rua Albano de Almeida, no․ 335, CEP 37410-000. Santa Rita de Cássia, Itajubá, Minas Gerais.

E-mail: enf_maiume@yahoo.com.br
Recebido: $30 / 08 / 2017$

Finalizado: 03/04/2018 


\section{INTRODUÇÃO}

Feridas crônicas (FC) são consideradas uma epidemia mundial ${ }^{(1)}$. Acometem mais a população idosa e representam altos custos para a saúde. Atingem cerca de $1 \%$ a $2 \%$ da população em geral com incidência de $0,76 \%$ em homens e 1,42\% nas mulheres ${ }^{(2-3)}$.

FC são definidas como uma ruptura na superfície da pele, incluindo uma ou mais camadas, com processo cicatricial lentificado ${ }^{(4)}$. Assim, para minimizar o tempo de cicatrização e, consequentemente, diminuírem os custos para os pacientes e familiares, o tratamento de feridas crônicas depende de um diagnóstico objetivo, fundamentado principalmente na avaliação da ferida e na escolha do agente terapêutico adequado ${ }^{(5-6)}$.

Nesse cenário, o enfermeiro está em destaque, pois é o profissional da saúde responsável pela assistência ao paciente com lesão. Sua função é elencar estratégias para a prevenção, a avaliação e o tratamento da ferida visando à reparação tecidual em um curto período de tempo para minimizar complicações e comprometimentos ${ }^{(7)}$.

Otratamento de feridas demanda do profissional, além de uma postura ética, uma base de conhecimento para avaliar a descontinuidade da pele e as condições do paciente, identificando os fatores que podem se opor à cicatrização e intervindo em cada fase do processo de reparação tecidual. Uma boa avaliação permite, ao enfermeiro, tomar decisões de forma segura para um tratamento eficiente ${ }^{(8)}$.

Todavia, a assistência ao paciente com ferida se torna eficaz quando todas as observações da avaliação e os resultados das intervenções prestadas são anotados e documentados ${ }^{(9)}$.

A disponibilização de um instrumento na língua portuguesa fornecerá uma ferramenta para a atuação dos profissionais da saúde, principalmente do enfermeiro, tanto na pesquisa investigativa, como, também, para o desenho de intervenções, além de nortear o profissional no âmbito dos cuidados prestados ao paciente com FC.

O instrumento RESVECH 2.0 (Resultados Esperados da Avaliação da Cicatrização de Feridas Crônicas) foi construído para avaliar o processo de cicatrização de FC (úlceras de membros inferiores e lesão por pressão). Na sua versão original, o instrumento RESVECH 2.0 é composto por seis domínios que caracterizam a reparação tecidual da ferida: dimensão, profundidade, bordas, tipos de tecido, exsudato e infecção/inflamação(10).

Cada item possui descrições sobre a apresentação da ferida no momento da avaliação obtendo um escore para cada item. Apenas o item infecção/inflamação possui subitens que são graduados individualmente. Os pontos atribuídos a cada domínio devem ser somados e o valor total, que pode variar de zero (ferida cicatrizada) a 35 (pior estado de cicatrização de feridas), será o escore final do instrumento ${ }^{(10)}$.

Obteve-se, na primeira avaliação, um Alfa de Cronbach de 0,72. Nas demais avaliações, o alfa permeou de 0,73 a 0,84, conferindo ao questionário confiabilidade. Observou-se, também, que este valor aumenta na medida em que a ferida evolui positivamente. O questionário apresentou correlação entre suas variáveis e demonstrou ser sensível às mudanças internas da amostra do estudo.

O estudo se justifica devido à importância no contexto profissional e social, uma vez que disponibilizará um questionário para os profissionais da saúde avaliarem a ferida crônica de forma sistematizada e objetiva e, nesse contexto, este estudo teve como objetivo traduzir e adaptar um questionário denominado RESVECH 2.0 por ser um instrumento que possibilita a prestação de cuidados com qualidade reduzindo de forma significativa o processo de reparação tecidual e, consequentemente, proporcionando, ao paciente, uma melhor qualidade de vida. 


\section{MÉTODO}

Estudo metodológico no qual foi realizada a tradução e a adaptação cultural do instrumento RESVECH 2.0 para a língua portuguesa brasileira. Os autores obtiveram aprovação do Comitê de Ética em Pesquisa da Universidade Vale do Rio Verde - Unincor sob o parecer n. ${ }^{\circ} 2.082 .722$ e declaração de consentimento do autor para o procedimento de tradução.

$\mathrm{Na}$ adaptação cultural do questionário "RESVECH 2.0" para o português, seguiram-se as etapas metodológicas recomendadas internacionalmente objetivando uma adaptação completa do instrumento, maximizando a obtenção semântica (para manter o mesmo significado de cada item após a tradução para o outro idioma), idiomática (busca expressões ou explicações correspondentes na língua-alvo, pois expressões idiomáticas não podem ser traduzidas), experiencial (avalia se os termos utilizados no instrumento são adequados à prática clínica na cultura do idioma onde a escala está sendo validada) e conceitual entre o instrumento original e o instrumento adaptado ${ }^{(11-15)}$.

A adaptação transcultural prevê cinco etapas: tradução inicial, síntese das traduções, retrotradução, avaliação por um comitê de especialistas e testagem da versão pré-final ${ }^{(12)}$.

Na primeira fase, tradução inicial, o instrumento RESVECH 2.0 foi enviado a dois tradutores independentes bilíngues cuja língua materna é a Língua Portuguesa. Na segunda fase, síntese das traduções, buscou-se a equivalência em relação ao instrumento original tanto de conteúdo, quanto cultural, semântica e conceitual, sendo as traduções efetuadas pelas pesquisadoras. Na terceira fase, foi realizada a retrotradução por dois tradutores com língua materna espanhola, porém, com domínio em Língua Portuguesa.

Na quarta fase, as duas versões, a traduzida e a retrotraduzida, como demonstrada na figura 1, foram comparadas por um comitê de especialistas com o objetivo de determinar as equivalências semântica, idiomática, cultural e conceitual entre o questionário original e a versão em português. Nessa fase, o comitê de especialistas produziu a versão final do instrumento para o pré-teste na língua portuguesa.

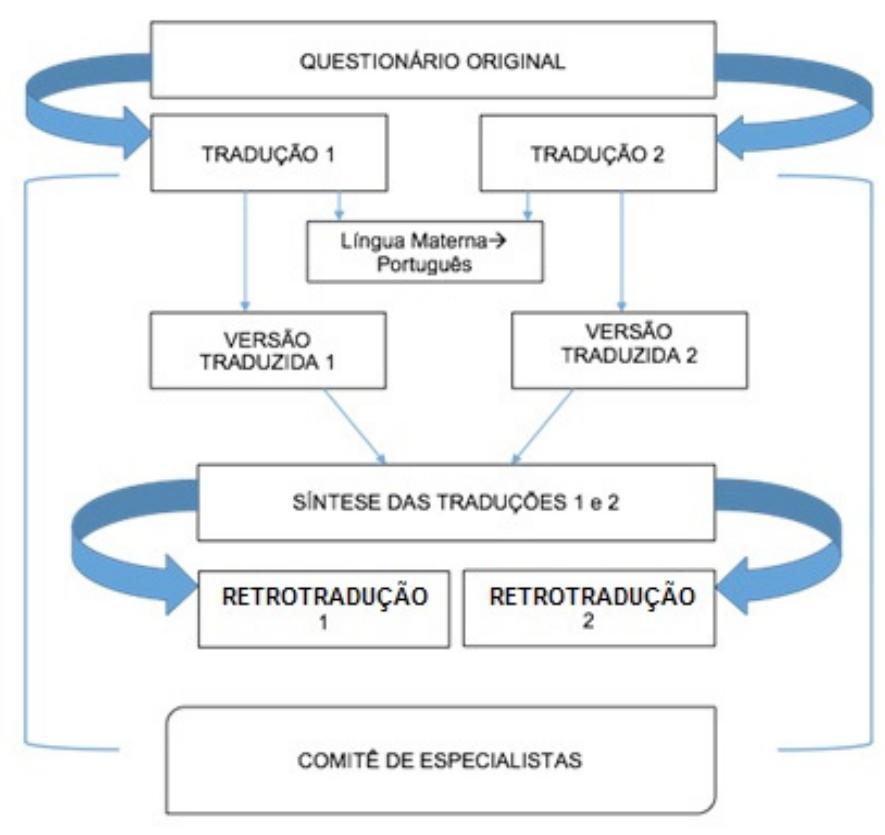

Figura 1- Guidelines for the process of cross-cultural adaptation of self-report measures, Spine, 2000

No que se refere à validade de conteúdo, o mesmo foi avaliado por meio do cálculo do Índice de Validade de Conteúdo (IVC), que avalia o nível de concordância entre os juízes sobre determinados aspectos do questionário adaptado e de seus itens ${ }^{(16)}$. 
Os juízes pontuaram os itens com valores de um a quatro, a saber: 1 - não equivalente; 2 - impossível avaliar a equivalência sem que o item seja revisto; 3 - equivalente, mas necessita de alterações menores e 4 - absolutamente equivalente $\mathrm{e}^{(16-17)}$.

O escore foi computado por meio da somatória dos itens que foram destacados com "3" e "4" dividindo-se o valor pelo número de juízes. Já os itens que receberam notas " 1 " e " 2 " foram revisados. Para a pesquisa, foi estipulado o nível de concordância igual ou superior a $0,8^{(16-17)}$.

Após as sugestões dos membros do comitê, obteve-se a versão pré-final do instrumento utilizada para o pré-teste. Essa versão foi aplicada a uma amostra por conveniência de 30 enfermeiros estomaterapeutas e/ou com prática clínica no tratamento de feridas de, no mínimo, 12 meses. Os instrumentos foram autoaplicados e os dados foram coletados individualmente em fevereiro de 2017.

Também foi solicitado, aos enfermeiros, que preenchessem uma ficha para a caracterização dos sujeitos (idade, sexo, ano de formação e especialização) e um questionário para a avaliação da praticabilidade do RESVECH 2.0.

O Questionário de Avaliação da Praticabilidade foi desenvolvido e validado no Brasil(16) e tem como objetivo verificar a facilidade com que os sujeitos respondem ao questionário e averiguar o tempo gasto para o preenchimento de instrumentos de medida ${ }^{(18)}$.

\section{RESULTADOS}

O processo de adaptação cultural foi realizado seguindo as etapas sistematicamente: tradução, síntese das traduções, retrotraduções e comitê de especialistas. Nelas, os resultados foram alcançados com êxito.

A tradução inicial foi realizada por um enfermeiro com conhecimento em feridas e um professor de línguas, de maneira independente, resultando em duas versões: versão traduzida um (VT1) e a versão traduzida dois (VT2).

A análise das divergências de ambas as versões foi realizada pelos dois tradutores juntamente com a pesquisadora. Assim, os resultados foram sintetizados resultando em uma versão traduzida, baseada no consenso, denominada Versão Traduzida um e dois - VT1T2.

$\mathrm{Na}$ etapa da retrotradução, ambos os tradutores não foram informados dos conceitos e objetivos da pesquisa, finalizando com duas traduções independentes. Em nenhuma das etapas, os tradutores relataram dificuldades nas traduções.

Em relação ao IVC, dois itens apresentam valores inferiores a 0,8, exsudato $(0,78)$ e bordas $(0,67)$, indicando que seriam necessárias revisões para que os mesmos atendessem às equivalências, como demonstra a tabela 1.

Tabela 1 - Índice de Validade de Conteúdo (IVC) entre os membros do Comitê de Especialistas. Itajubá, MG, Brasil, 2017. (continua)

\begin{tabular}{|c|c|c|c|c|c|c|c|c|c|c|c|}
\hline Equivalência Semântico-idiomática, conceitual e cultural & & & & & $\mathrm{mb}$ & & & & & T3/4* & IVC** \\
\hline Juízes & 1 & 2 & 3 & 4 & 5 & 6 & 7 & 8 & 9 & & \\
\hline Dimensões da ferida & 4 & 4 & 3 & 4 & 4 & 4 & 4 & 4 & 4 & 9 & 1 \\
\hline Profundidade/tecidos afetados & 3 & 3 & 4 & 3 & 3 & 3 & 4 & 3 & 4 & 9 & 1 \\
\hline Bordo & 3 & 3 & 3 & 3 & 3 & 2 & 1 & 3 & 1 & 6 & 0,67 \\
\hline Tipo de tecido no leito da ferida & 3 & 3 & 4 & 3 & 3 & 4 & 4 & 3 & 2 & 8 & 0,89 \\
\hline Exsudato & 3 & 3 & 4 & 3 & 4 & 2 & 1 & 3 & 3 & 7 & 0,78 \\
\hline Infecção/inflamação & 4 & 4 & 4 & 4 & 4 & 3 & 4 & 4 & 4 & 9 & 1 \\
\hline
\end{tabular}




\begin{tabular}{llllllllllll}
\hline Abrangência & 3 & 4 & 4 & 3 & 3 & 3 & 3 & 3 & 3 & 9 & 1 \\
Relevância & 4 & 4 & 4 & 3 & 3 & 4 & 3 & 4 & 3 & 9 & 1 \\
\hline
\end{tabular}

*T3 e 4= Total de respostas 3 e $4 .{ }^{* *}$ IVC $=$ Índice de Validade de Conteúdo

Assim, modificações foram realizadas de acordo com as sugestões dos juízes. Para tal, não foi necessária uma reunião presencial com o comitê de especialistas, uma vez que as colocações para as alterações foram similares entre o comitê e descritas pontualmente.

O item "borda" foi alterado pela palavra "margem", mais amplamente utilizada na cultura brasileira, e todas as suas descrições foram mantidas. Contudo, foi solicitado que se explicasse sucintamente e pontualmente cada característica. Já o item "exsudato" foi modificado de acordo com a cultura brasileira na qual os profissionais da saúde avaliam sua quantidade como pequena, média e grande, de acordo com as sugestões dos juízes.

Apesar de o item "profundidade" e o item "tipo de tecido no leito lesionar" apresentarem IVC adequados, dois juízes sugeriram pequenas modificações para auxiliarem no processo de avaliação de ferida, as quais foram aceitas. $\mathrm{Na}$ "profundidade", foi acrescentada a palavra "epitelização" na primeira característica e "no leito lesionar" foram descritos os tipos de necrose.

Os resultados das avaliações do comitê de especialistas demonstraram equivalência semânticoidiomática, cultural e conceitual entre o instrumento original e as traduções e retrotraduções. O instrumento final, com todas as alterações sugeridas pelos juízes, foi encaminhado para o autor para a sua aprovação e elas foram todas aceitas.

Após as alterações, a versão pré-final do instrumento foi submetida ao pré-teste para a avaliação da compreensão dos sujeitos. Participaram dessa etapa 30 enfermeiros, com média de idade de 35,9 anos, sendo $28(93,3 \%)$ do sexo feminino e dois $(6,7 \%)$ do sexo masculino. Vinte e um (70\%) tinham mais que dez anos de formados. Quanto à qualificação profissional, 26 (86,7\%) possuíam especialização; três enfermeiros tinham titulação de mestre e um estava cursando o doutorado.

Tabela 2 - Praticabilidade da versão brasileira do instrumento RESVECH 2.0 segundo participantes do pré-teste de avaliação de compreensão. Itajubá, MG, Brasil, 2017

\begin{tabular}{|c|c|c|c|c|c|c|c|c|c|c|c|c|}
\hline \multirow{2}{*}{ Questões } & \multicolumn{2}{|c|}{ DT } & \multicolumn{2}{|c|}{ DP } & \multicolumn{2}{|c|}{ NTO } & \multicolumn{2}{|c|}{$\mathrm{CP}$} & \multicolumn{2}{|c|}{$\mathrm{CT}$} & \multicolumn{2}{|c|}{ Total } \\
\hline & $\mathbf{N}$ & $\%$ & $\mathbf{N}$ & $\%$ & $\mathbf{N}$ & $\%$ & $\mathbf{N}$ & $\%$ & $\mathbf{N}$ & $\%$ & $\mathbf{N}$ & $\%$ \\
\hline 1. Achei fácil entender as instruções do questionário. & & & & & & & 9 & 30 & 21 & 70 & 30 & 100 \\
\hline 2. Achei fácil entender as questões do questionário. & & & & & & & 8 & 26,7 & 22 & 73,3 & 30 & 100 \\
\hline 3. Achei fácil assinalar as respostas do questionário. & & & & & & & 9 & 30 & 21 & 70 & 30 & 100 \\
\hline
\end{tabular}

DT - Discordo totalmente; DP - Discordo parcialmente; NTO - Não tenho opinião; CP - Concordo parcialmente; CT - Concordo totalmente.

Durante o pré-teste, três sujeitos (10\%) ficaram em dúvida quanto ao item cinco referente ao "exsudato seco ou úmido". Uma enfermeira referiu "não sei o que é exsudato seco, pois se está exsudando alguma coisa tem que estar úmido". Outro enfermeiro sugeriu que ficaria um pouco mais clara a questão se ficasse "sim ou não, ao invés de seco ou úmido". E outra também questionou: "Exsudato seco? Para mim, tem ou não exsudato". Por isso, apesar da maioria dos sujeitos não referir dificuldade de entendimento no item, ele não sofreu modificações após a aplicação do pré-teste.

Quanto ao entendimento dos outros itens do questionário, apenas uma enfermeira referiu não saber o que é "tecido compatível com biofilme" e que nunca viu uma "hipergranulação".

Quanto à praticabilidade do instrumento, o tempo médio de preenchimento do RESVECH foi de três minutos. A maioria das respostas dos itens foi a opção concordo totalmente, conforme a tabela 2. Vinte e um $(70 \%)$ enfermeiros relataram que as questões foram facilmente compreendidas e $21(70 \%)$ avaliaram 
como de fácil preenchimento os itens do instrumento. A maioria dos sujeitos $(22=73,3 \%)$ considerou os itens de fácil entendimento.

\section{DISCUSSÃO}

A assistência ao paciente com feridas deve ser baseada em um cuidado integral e a avaliação da ferida é parte fundamental desse processo ${ }^{(19)}$. Para nortear os profissionais na avaliação, são fundamentais a utilização de instrumentos que lhes fornecerão subsídios no tratamento de feridas e a escolha do agente terapêutico adequado, além de possibilitar o acompanhamento da evolução da lesão durante todo o processo de reparação tecidual em cada fase da cicatrização ${ }^{(20)}$.

Nesse contexto, a disponibilização de um instrumento para a cultura brasileira auxiliará na assistência de qualidade ao paciente com ferida. Distintos estudos têm utilizado o instrumento RESVECH 2.0 no controle de avaliação da ferida ${ }^{(21)}$.

Um estudo realizado na Espanha buscou determinar a qualidade de vida relacionada à saúde (QVRS) em pacientes que sofrem de ulceração venosa e correlacionar o estado de gravidade da ferida com a perda de QVRS, bem como identificar os aspectos da QVRS mais afetados negativamente pela presença de úlceras venosas. Os dados foram coletados por um período de três a cinco meses. Foi utilizada a escala RESVECH 2.0 para monitorar feridas. A escala MAID foi utilizada para medir a gravidade da ferida. O questionário sobre a úlcera venosa cruzada Charing Cross (CCVUQe) (versão em espanhol) foi utilizado para avaliar a qualidade de vida. Os dados evidenciaram que as úlceras venosas afetam a QVRS dos pacientes e, particularmente, seu estado emocional. Existe uma relação entre a gravidade da ferida e a perda de QVRS. A presença de tecido não viável, o controle de exsudato e a infecção determinam a perda de QVRS ${ }^{(22)}$.

Outro estudo, realizado em Portugal, objetivou avaliar o perfil clínico de uma amostra de indivíduos com FC em uma instituição oncológica, por meio da escala RESVECH 2.0, além de caracterizar o perfil sociodemográfico de um doente oncológico com FC, validar o instrumento de trabalho RESVECH 2.0 e avaliar a qualidade de vida (QV) desses indivíduos. Os resultados da validação demonstram a escala com critérios de fiabilidade, registrando-se uma boa consistência interna, com um alfa de Cronbach para o primeiro observador de 0,735 e, para o segundo observador, de 0,741. Obteve-se, também, um coeficiente de correlação intercalasses (ICC) muito forte de 0,979. Para os itens dicotômicos da escala, obteve-se um coeficiente de concordância de Kappa que varia entre 0,78 e 0,96. Concluiu-se que a escala RESVECH 2.0 é uma ferramenta eficaz que representa um instrumento de avaliação e registro para o controle das $\mathrm{FC}^{(23)}$.

Neste estudo, a tradução e a adaptação do questionário de avaliação de feridas seguiram as recomendações internacionais de acordo com o processo de tradução, síntese da tradução, retrotradução e avaliação pelo comitê de especialistas ${ }^{(10)}$. Essas etapas foram seguidas sistematicamente e sem intercorrências resultando em uma tradução fidedigna ao instrumento original.

A utilização de normas metodológicas internacionalmente aceitas para a realização da adaptação cultural facilita a reprodutibilidade dos resultados e possibilita a comparação entre diferentes populações ${ }^{(10)}$.

A adaptação cultural do RESVECH 2.0 teve como finalidade encontrar equivalência entre a versão original do instrumento e a versão brasileira, uma vez que esse processo é complexo. O termo "adaptação cultural" é utilizado para abranger um processo que analisa a língua/tradução durante a elaboração de um questionário para uso em outro país ${ }^{(10)}$. O pesquisador deve considerar não só o idioma, mas, também, as diferenças culturais de percepção de saúde daquela região, do contexto cultural e do estilo de vida da população em questão(24). Dessa maneira, é possível manter a equivalência linguística e cultural dos itens de um instrumento mantendo sua relevância mesmo que seja necessário modificá-los para a cultura daquele lugar ${ }^{(25)}$.

Deve-se reconhecer que os itens não precisam ser somente traduzidos bem linguisticamente, mas, também, ser adaptado culturalmente para manter a validade de conteúdo do instrumento em diferentes 
culturas $^{(11)}$. Dessa forma, pode-se descrever, de maneira mais fidedigna, o impacto de uma doença ou de seu tratamento de forma semelhante em ensaios multicêntricos ou multinacionais.

Referente à validade de conteúdo, o comitê de especialistas é uma etapa fundamental para obter as equivalências interculturais ${ }^{(11)}$. Assim, as sugestões dos juízes foram essenciais para adequar o instrumento para a sua utilização no cenário brasileiro. Todas as recomendações dos especialistas foram acatadas, mesmo quando o item avaliado apresentou índice de validade de conteúdo satisfatório, uma vez que as colocações permitiram elucidar as questões resultando em um instrumento de fácil compreensão e aplicação.

A validação de conteúdo é uma etapa fundamental, pois está relacionada aos julgamentos a respeito do instrumento. Tais julgamentos são realizados por distintos especialistas no assunto que possuem como responsabilidade analisar a representatividade e relevância das questões referentes ao conteúdo que se deseja mensurar ${ }^{(25)}$. No processo de adaptação, o comitê de juízes também tem como função avaliar se os itens correspondem à realidade cultural ${ }^{(26)}$.

Todas as adaptações realizadas no instrumento final foram encaminhadas ao autor para apreciação e aprovação, o que reforça a autenticidade do instrumento em relação ao original.

No pré-teste do instrumento, realizado com 30 enfermeiros, foi evidenciada uma boa aceitação e facilidade de compreensão. $O$ fato de três participantes terem apresentado apenas uma dúvida quanto ao entendimento do item "exsudato" do instrumento indica que o rigor metodológico deste estudo foi executado possibilitando a tradução e a adaptação cultural adequadas e dentro das normas vigentes. A adoção de uma linguagem clara, simples e de fácil acesso também possibilitou o rápido entendimento do instrumento pelos sujeitos da pesquisa.

Apesar de uma enfermeira não saber o que significava o termo "biofilme" e "hipergranulação" durante a avaliação do questionário, foi explicado para a mesma, na prática, o que significa cada termo utilizado para a avaliação de feridas.

As avaliações realizadas pelo comitê de especialistas e durante o pré-teste contribuíram para essa facilidade na compreensão dos itens do instrumento e permitiram avaliar, na prática, a aplicação do instrumento.

\section{CONCLUSÃO}

O instrumento RESVECH 2.0 foi adaptado para a cultura brasileira com êxito seguindo as etapas recomendadas pela literatura internacional.

Espera-se que a adaptação cultural do RESVECH 2.0 possa facilitar o processo de avaliação de feridas pelos profissionais da área de saúde e que o instrumento possa ser utilizado em outras pesquisas para a avaliação de qualidade de vida correlacionando com uma medida objetiva.

Recomenda-se estudo de validação do instrumento a fim de verificar suas propriedades de medida.

Quadro 1 - Características do Comitê de Especialistas. Itajubá, MG, Brasil, 2017. (continua)

\begin{tabular}{|c|c|c|}
\hline Juízes & Profissão & Experiência \\
\hline 01 & Enfermeiro (a), mestre em Enfermagem & $\begin{array}{r}\text { Professor universitário com experiência na área assistencial } \\
\text { em tratamento de feridas. }\end{array}$ \\
\hline 02 & Enfermeiro (a), doutor (a) em Enfermagem & $\begin{array}{c}\text { Professora universitária com experiência em metodologia da } \\
\text { pesquisa. }\end{array}$ \\
\hline 03 & Professor (a) & Formado em Línguas. \\
\hline 04 & Enfermeiro (a), doutor (a) em Enfermagem & $\begin{array}{c}\text { Experiência na assistência aos pacientes com feridas e } \\
\text { estudos envolvendo qualidade de vida e pessoas idosas; } \\
\text { adaptação e validação de instrumentos. }\end{array}$ \\
\hline
\end{tabular}




\begin{tabular}{|c|c|c|}
\hline 05 & Enfermeiro (a), mestre em Enfermagem & $\begin{array}{l}\text { Professora universitária com experiência na área assistencial } \\
\text { e estudos envolvendo feridas e comunicação terapêutica. }\end{array}$ \\
\hline 06 & Enfermeiro (a), Estomaterapeuta & $\begin{array}{l}\text { Professora universitária com experiência na área assistencial } \\
\text { e em estudos envolvendo feridas, incontinência e estomas. }\end{array}$ \\
\hline 07 & Enfermeiro (a), mestre em Enfermagem, & $\begin{array}{c}\text { Professora universitária com experiência em metodologia da } \\
\text { pesquisa. }\end{array}$ \\
\hline 08 & $\begin{array}{l}\text { Enfermeiro (a), gerente em unidade de } \\
\text { tratamento de feridas }\end{array}$ & $\begin{array}{l}\text { Professor universitário com experiência na área assistencial } \\
\text { em tratamento de feridas. }\end{array}$ \\
\hline 09 & $\begin{array}{l}\text { Enfermeiro (a), Estomaterapeuta, } \\
\text { responsável por um ambulatório e feridas }\end{array}$ & Experiência na área assistencial em tratamento de feridas. \\
\hline
\end{tabular}

\section{- REFERÊNCIAS}

1. Steinberg J, Siddiqui F. The Chronic Wound and the Role of Biofilm. Podiatry Management. [Internet] 2011;30(6):18190 [acesso em 06 jun 2017]. Disponível: http://podiatrym.com/cme/CME811.pdf.

2. Margolis DJ, Bilker W, Santanna J, Baumgarten M. Venous leg ulcer: Incidence and prevalence in the elderly. J. Am. Acad. Dermatol. [Intenet] 2002;46(3):381-86 [acesso em 08 jun 2017]. Disponível: https://doi.org/10.1067/ mjd.2002.121739.

3. Grundmann RT. Chronic and surgical wounds healing by secondary intention. Epidemiology and review on clinical effectiveness and cost-effectiveness of dressings and wound management. Chirurgische Praxis. 2010;72(4):573-598.

4. Lawall H. Treatment of chronic wounds. Vasa. [Internet] 2012;41:396-409 [acesso em 08 jun 2017]. Disponível: https://doi.org/10.1024/0301-1526/a000230.

5. de Mendonça RJ, Coutinho-Netto J. Aspectos celulares da cicatrização. An. Bras. Dermatol. [Internet] 2009;84(3):25762 [acesso em 08 jun 2017]. Disponível: http://dx.doi.org/10.1590/S0365-05962009000300007.

6. Stremitzer S, Wild T, Hoelzenbein T. How precise is the evaluation of chronic wounds by health care professionals? International Wound Journal. [Internet] 2007;4(2) [acesso em 05 jun 2017]. Disponível: http://dx.doi.org/10.1111/j.1742481X.2007.00334.x.

7. Morais GFC, Oliveira SHS, Soares MJGO. Avaliação de feridas pelos enfermeiros de instituições hospitalares da rede pública. Texto contexto-nferm. [Internet] 2008;17(1):98-105 [acesso em 05 jun 2017]. Disponível: http://dx.doi. org/10.1590/S0104-07072008000100011.

8. dos Santos AAR, Medeiros ABA, Soares MJGO, Costa MML. Avaliação e tratamento de feridas: o conhecimento de acadêmicos de enfermagem. Rev. enferm. UERJ. [Internet] 2010;18(4):547-52 [acesso em 3 jul 2017]. Disponível: http://www.facenf.uerj.br/v18n4/v18n4a08.pdf.

9. Shepherd J, Nixon M. Standardising wound care documentation in clinical practice: The wound healing assessment and monitoring (WHAM) tool. Wounds UK. [Internet] 2013;9(1) [acesso em 05 jun 2017]. Disponível: https://www. wounds-uk.com/journals/issue/33/article-details/standardising-wound-care-documentation-in-clinical-practice.

10. Restrepo-Medrano JC, Soriano JV. Development of a wound healing index for chronic wounds. Gerokomos. [Internet] 2011;22(4):176-83 [acesso em 10 fev 2017]. Disponível: http://dx.doi.org/10.4321/S1134-928X2011000400005.

11. Beaton D, Bombardier C, Guillemin F, Ferraz MB. Recommendations for the Cross-Cultural Adaptation of the DASH \& QuickDASH Outcome Measures. Institute for Work and Health. [Internet] 2007 [acesso em 26 jun 2013]. Disponível: http://www.dash.iwh.on.ca/sites/dash/files/downloads/cross_cultural_adaptation_2007.pdf.

12. Guillemin F. Cross-cultural adaptation and validation of health status measures. Scand. J. Rheumatol. [Internet] 1995;24(2):61-3 [acesso em 10 jul 2017]. Disponível: https://doi.org/10.3109/03009749509099285. 
13. Beaton DE, Bombardier C, Guillemin F, Ferraz MB. Guidelines for the process of cross-cultural adaptation of selfreport measures. Spine. [Internet] 2000;25(24):3186-91 [acesso em 10 jul 2017]. Disponível: https://journals.Iww.com/ spinejournal/Citation/2000/12150/Guidelines_for_the_Process_of_Cross_Cultural.14.aspx.

14. Alexandre NMC, Guirardello EB. Adaptación cultural de instrumentos utilizados en salud ocupacional. Rev. Panam. Salud Publica. [Internet] 2002;11(2):109-11 [acesso em 11 jul 2017]. Disponível: http://dx.doi.org/10.1590/S102049892002000200007.

15. Consensus-based Standards for the selection of health Measurement Instruments (COSMIN). COSMIN Checklist manual. [internet] Amsterdam: COSMIN; 2012 [acesso em 30 ago 2017]. Disponível: http://www.cosmin.nl/cosmin_ checklist.html.

16. Alexandre NMC, Coluci MZO. Validade de conteúdo nos processos de construção e adaptação de instrumentos de medida. Ciênc. Saúde Coletiva. [Internet] 2011;16(7) [acesso em 10 jul 2017]. Disponível: http://www.scielo.br/scielo. php?pid=S1413-81232011000800006\&script=sci_abstract\&tlng=pt.

17. Devon HA, Block ME, Moyle-Wright P, Ernst DM, Hayden SJ, Lazzara DJ, et al. A psychometric toolbox for testing validity and reliability. Journal of Nursing Scholarship. [Internet] 2007;39(2):155-64 [acesso em 10 jul 2017]. Disponível: https://doi.org/10.1111/j.1547-5069.2007.00161.x.

18. Coluci MZO, Alexandre NMC. Desenvolvimento de um questionário que avalia a praticabilidade de instrumentos de medidas. Rev. enferm. UERJ. [Internet] 2009;17(3):378-82 [acesso em 10 jul 2017]. Disponível: http://www.facenf. uerj.br/v17n3/v17n3a14.pdf.

19. Greatrex-White S, Moxey H. Wound assessment tools and nurses' needs: an evalution study. International Wound Journal. [Internet] 2015;12(3):293-301 [acesso em 11 jul 2017]. Disponível: https://doi.org/10.1111/iwj.12100.

20. Espirito Santo PF, de Almeida SA de, Silveira MM, Salomé GM, Ferreira LM. Uso da ferramenta Pressure Ulcer Scale for Healing para avaliar a cicatrização de úlcera crônica de perna. Rev. Bras. Cir. Plást. [Internet] 2013;28(1):133:41 [acesso em 20 jun 2017]. Disponível: http://dx.doi.org/10.1590/S1983-51752013000100023.

21. Folguera-Álvarez C, Garrido-Elustondo S, Verdú-Soriano J. et al. ECAMulticapa: Effectiveness of double-layered compression therapy for healing venous ulcers in primary care: a Study Protocol. BCM Nursing. [Internet] 2016;15(58) [acesso em 20 jun 2017]. Disponível: https://doi.org/10.1186/s12912-016-0179-x.

22. González de La Torre H, Quintana-Lorenzo ML, Perdomo-Pérez E, Verdú J. Correlation between health-related quality of life and venous leg ulcer's severity and characteristics: a cross-sectional study. International Wound Journal. [Internet] 2017;14(2):360-68 [acesso em 20 jun 2017]. Disponível: https://doi.org/10.1111/iwj.12610.

23. Lourenço MCP. Avaliação da ferida crônica [dissertação]. Coimbra (PT): Faculdade de Economia da Universidade de Coimbra; 2016. Disponível: http://hdl.handle.net/10316/32784.

24. Guillemin F, Bombardier C, Beaton D. Cross-cultural adaptation of health-related quality of life measures: Literatre review and proposed guidelines. J. Clin. Epidemiol. [Internet] 1993;46(12):1417-32 [acesso em 20 jun 2017]. Disponível: https://doi.org/10.1016/0895-4356(93)90142-N.

25. Raymundo VP. Construção e validação de instrumentos: um desafio para a psicolinguística. Letras de Hoje, Porto Alegre. [Internet] 2009;44(3):86-93 [acesso em 20 jun 2017]. Disponível: http://revistaseletronicas.pucrs.br/ojs/index. php/fale/article/view/5768/4188.

26. Alexandre NMC, Coluci MZO. Validade de conteúdo nos processos de construção e adaptação de instrumentos de medidas. Ciências \& Saúde Coletiva. [Internet] 2011;16(7) [acesso em 20 jun 2017]. Disponível: http://dx.doi. org/10.1590/S1413-81232011000800006. 